\title{
Mitigating Channel Estimation Error with Timing Synchronization Tradeoff in Cooperative Communications
}

\author{
Ahmed S. Ibrahim $\dagger$ and K. J. Ray Liu $\ddagger$ \\ $\dagger$ Department of Signals and Systems \\ Chalmers University of Technology, SE-412 96 Gothenburg, Sweden \\ asibrahim@ieee.org \\ $\ddagger$ Department of Electrical and Computer Engineering \\ University of Maryland, College Park, MD 20742, USA \\ kjrliu@umd.edu
}

\begin{abstract}
Channel estimation error and co-channel interference (CCI) problems are among the main causes of performance degradation in wireless networks. In this paper, we investigate the impact of cooperative communications on mitigating the effect of channel estimation error and CCI. Two main performance criteria, namely, the traditional outage probability and the proposed signal-tonoise ratio (SNR) gap ratio, are utilized to characterize such impact. The SNR gap ratio measures the reduction in the SNR due to channel estimation error or CCI. Taking into consideration the channel estimation error, we show that the outage probability is reduced by utilizing cooperative transmission protocols. We also show that cooperative transmission scenarios, in which each cooperating relay forwards its signal over an orthogonal channel, result in lower SNR gap ratio compared to that of the direct transmission. Thus, cooperative transmission schemes are less susceptible to the effect of channel estimation error compared to direct transmission. Moreover, increasing the number of cooperating relays reduces the effect of the channel estimation error more. Timing synchronization error arises in distributed space-time cooperative schemes, in which the cooperating relays are simultaneously transmitting their signals over the same channel. Unlike the channel estimation error, the effect of the timing synchronization error gets worse as the the number of cooperating relays increases. In this work we also study the tradeoff between the timing synchronization error and the channel estimation error, and show their net impact on the system performance. Finally, we illustrate that CCI can be modeled in a similar fashion to the channel estimation error, and hence the cooperative transmission schemes are also less susceptible to the effect of CCI.
\end{abstract}

Keywords: Channel estimation error, co-channel interference, cooperative diversity, distributed transmit beamforming, relay selection, timing synchronization error. 


\section{Introduction}

Recently, cooperative communications for wireless networks have gained much interest due to its ability to mitigate fading in wireless networks through achieving spatial diversity, while resolving the difficulties of installing multiple antennas on small communication terminals. Cooperative communication protocols make use of the broadcast nature of wireless channels, where a number of relay nodes are assigned to help a source in forwarding its information to its destination, hence forming a virtual antenna array. Various cooperative diversity protocols were proposed and analyzed in $[1,2,3,4,5,6,7,8]$.

In [2], Laneman et al. described various techniques of cooperative communication such as decodeand-forward and amplify-and-forward. In [3], it was shown that there exists a collaborative code that can achieve almost the same performance gain as that of traditional space-time codes. The symbol error rate (SER) for single- and multi-node decode-and-forward cooperative technique was analyzed in $[4,5]$. It was shown that the conventional cooperative communication scenario, in which each of the available $N$ relays forwards the source's information over an orthogonal channel (e.g. time or frequency slot), achieves full diversity order equal to $N+1$. However, the bandwidth efficiency drops to $1 /(N+1)$ symbols per channel use (SPCU). In [6] a relay selection cooperative scheme, in which only one relay forwards the source's information, has been proposed. It was shown that relay-selection cooperative scheme achieves high bandwidth efficiency while guaranteeing full diversity order. Distributed space-time cooperative schemes, in which the helping relays simultaneously forward modified version of the source's information, have been proposed and analyzed in $[7,8]$. It was shown that distributed cooperative schemes, under certain conditions, can achieve full diversity order.

Channel estimation error, caused possibly by Doppler shift or noise on the pilot signals, can cause dramatic performance degradation in wireless networks. In [9], it was examined that channel estimation error results in lower average signal-to-noise ratio (SNR) and higher average error rate in orthogonal frequency division multiplexing (OFDM)-based systems. It was also shown in [10] via simulations results that channel estimation error causes error floor in the amplify-and-forward 
cooperative scheme. In [11], a superposition coding scheme was proposed to reduce the channel estimation effect when the users have largely different SNR.

In addition to the channel estimation error problem, the co-channel interference (CCI) problem also arises in networks such as cellular networks, in which users of different neighboring cells are simultaneously transmitting their data over the same channels (e.g. OFDM subcarriers). CCI results in lower signal to interference plus noise ratio (SINR), which causes dramatic performance degradation. Recently, there have been some works that studied the impact of the multiple-input multiple-output (MIMO) techniques on the CCI problem $[12,13,14,15]$, in which it was shown that MIMO techniques can reduce the effect of the CCI problem. In general, we note that in communication systems with channel estimation error or CCI, we cannot get arbitrarily large SNR for high transmission power.

Motivated by the bad impact of channel estimation error and CCI on the direct transmission scenario, we investigate in this paper the ability of the various cooperative transmission protocols mentioned above to mitigate such impact. We consider two main performance criteria to characterize the impact of cooperative communications on channel estimation error, namely, the traditional outage probability and the proposed SNR gap ratio. The SNR gap ratio quantifies the reduction in the SNR due to channel estimation error. First, we show that the outage probability is reduced due to utilizing cooperative communication scenarios in the presence of channel estimation error. Second, we illustrate that cooperative transmission protocols, either the conventional or the relay-selection schemes, reduce the SNR gap ratio compared to that of the direct transmission. We find that cooperative communication protocols are less susceptible to channel estimation error by achieving spatial diversity via relays and distributing the total transmission power across multiple transmission phases. Moreover, increasing the number of relays reduces the effect of the channel estimation error more. With respect to CCI, we also show that cooperative communication protocols can mitigate the effect of CCI problem compared to the direct communication.

Unlike the conventional and relay-selection cooperative protocols, distributed space-time cooperative schemes allow simultaneous transmission among the cooperating relays. In these schemes, 
there is no guarantee that all the cooperating relays start their transmission at the same instant since they are not completely synchronized with each other. Furthermore, the received signals at the destination from the simultaneously transmitting relays experience different propagation delays. Therefore distributed space-time cooperative schemes suffer from timing synchronization error, which results in interference terms that dramatically increase the error rate $[16,17]$. Unlike the channel estimation error, increasing the number of relays increases the timing synchronization error effect. In this paper, we study the tradeoff of the impact of the channel estimation and the timing synchronization errors on the performance of the distributed transmit beamforming cooperative scheme. For a fixed channel estimation error variance, we show that at low data transmission power the effect of the timing synchronization error is more significant, and having more relays results in higher SNR gap ratio. As the transmission power increases, the channel estimation error dominates and having more relays leads to lower SNR gap ratio.

In this work, we focus on the single-carrier analysis, however, the analysis can be easily extended to the multi-carrier OFDM systems. The rest of this paper is organized as follows. In the next section, we describe the system model of the communication system, taking into consideration the channel estimation error effect, and explain the problem formulation. We Study the impact of various communication scenarios on the channel estimation error and CCI in Section 3. In Section 4, we consider the timing synchronization error and how it interacts with the channel estimation error. Finally, Section 5 concludes the paper.

\section{System Model and Problem Formulation}

Communication scenarios, which are based on training sequences (pilots) for channel estimation, are implemented in two consecutive phases, namely, training phase and data transmission phase. In the training phase, the channel is estimated using a known training sequence with a particular pilot power, denoted by $P_{\text {pilot }}$. A particular pilot transmission power $P_{\text {pilot }}$ results in a certain level of channel estimation error variance, referred to as $\alpha$, which is inversely proportional to the pilot transmission power. In the end of the training phase, the receiver has an estimate of the channel 


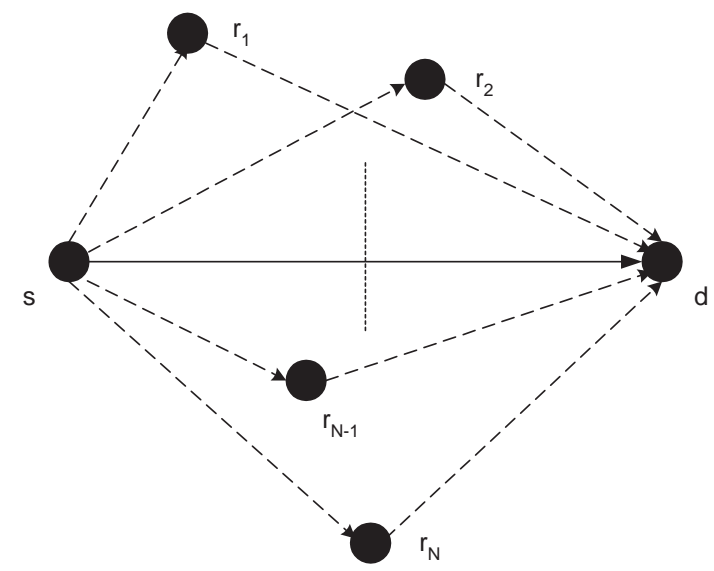

Figure 1: Cooperative communication system with a set of $N$ relays. Solid line represents the direct transmission and dashed lines represent the cooperative transmissions via the relays.

to be utilized in the coherent detection of the transmitted data in the following data transmission phase. In the data transmission phase, the channel estimate is fixed and does not depend on the data transmission power, $P$. Hence, the channel estimation error does not depend on the data transmission power, $P$.

The communication system under consideration is shown in Figure 1. It consists of the source, $s$, the destination, $d$, and a set of $N$ transmitting/receiving nodes, $r_{1}, r_{2}, \cdots, r_{N}$, which will be referred to as relays. We note that each of the nodes $s, r_{i}, i=1,2, \cdots, N$ has a data of its own, and its role interchanges between being a source sending its own information or a relay forwarding other nodes information. We assume that all the $N+1$ transmitting nodes utilize the same pilot transmission power, $P_{\text {pilot }}$, to allow the destination to estimate the corresponding channel responses. Each node broadcasts its pilot signal in a separate training phase. The transmitted pilot signal is received by the destination as well as all the other nodes. Every receiving node can then estimate its corresponding channel response with the sender. We note that no extra pilot transmission power is needed in the cooperative transmission protocols compared to that required in the direct transmission case. Finally, we assume that there is a fixed channel estimation error variance, $\alpha$, resulting from the training phase due to utilizing pilot transmission power of $P_{\text {pilot }}$.

We take into consideration the channel estimation error in the data transmission phase as follows. In the direct transmission scenario, the source sends its data symbol to the destination in one phase, 
which can be a time or frequency slot. The received symbol at the destination can be modeled as

$$
y_{s, d}^{D}=\sqrt{P}\left(h_{s, d}+h_{\alpha}\right) x+\sqrt{N_{0}} \eta_{\alpha},
$$

where the superscript $D$ denotes the direct transmission scenario, $x$ is the transmitted symbol with unit energy, i.e., $|x|^{2}=1, h_{s, d}$ is the estimated source-destination channel coefficient, $h_{\alpha}$ denotes the channel estimation error, $\eta_{\alpha}$ is a zero-mean additive white Gaussian noise (AWGN) with unit variance, and $N_{0}$ is the noise variance. In [11], the additional term resulting from channel estimation error, namely $\sqrt{P} x h_{\alpha}$ in (1), was called self-noise because it represents an added noise term that scales with the data transmission power.

The channel estimation error is a summation of large number of small quantities representing the inter-carrier interference and noise, and hence it can be modeled as a Gaussian random variable via the central limit theorem [18]. Similar to [9], the channel estimation error $h_{\alpha}$ is modeled as a zero-mean complex Gaussian random variable with variance $\alpha$. For constant modulus transmitted symbol $x$, the additional self-noise term $\left(\sqrt{P} x h_{\alpha}\right)$ is a zero-mean complex Gaussian random variable with variance $\alpha P$. Thus, (1) can be rewritten as

$$
y_{s, d}^{D}=\sqrt{P} h_{s, d} x+\sqrt{\alpha P+N_{0}} \eta_{s, d},
$$

where $\eta_{s, d}$ is a zero-mean Gaussian random variable with unit variance. We note that the system model in (2) is similar to the one that was considered to represent the channel estimation error effect in $[11]$.

In the conventional $N$-relay cooperative transmission scenario, a transmission of one symbol is implemented in $N+1$ phases. In the first phase, the source broadcasts its symbol to the relays and the destination with a transmission power of $P_{0}$. Taking into consideration the channel estimation error as in (2), the received symbols at the destination and the $i$-th relay can be modeled as

$$
\begin{aligned}
& y_{s, d}^{C}=\sqrt{P_{0}} h_{s, d} x+\sqrt{\alpha P_{0}+N_{0}} \eta_{s, d}, \\
& y_{s, r_{i}}^{C}=\sqrt{P_{0}} h_{s, r_{i}} x+\sqrt{\alpha P_{0}+N_{0}} \eta_{s, r_{i}}, \quad i=1,2, \cdots, N .
\end{aligned}
$$

respectively, where the superscript $C$ denotes the cooperative transmission scenario, $h_{s, r_{i}}$ is the estimated channel coefficient between the source and the $i$-th relay, and $\eta_{s, r_{i}}$ is a zero-mean AWGN with unit variance. 
In this paper, we consider the decode-and-forward cooperative protocol $[4,5]$. In the decode-andforward protocol, each relay decides whether to forward the received information or not according to the quality of the received signal. We assume that every relay can tell whether the received information is correctly decoded or not $[4,5]$. If the $i$-th relay correctly decodes the received symbol, then it forwards the decoded symbol to the destination in the $(i+1)$-th phase, otherwise it remains idle. The received symbol at the destination in the $(i+1)$-th phase is given by

$$
y_{r_{i}, d}^{C}=\sqrt{\tilde{P}_{i}} h_{r_{i}, d} x+\sqrt{\alpha \tilde{P}_{i}+N_{0}} \eta_{r_{i}, d},
$$

where $\tilde{P}_{i}=P_{i}$ if the relay decodes the symbol correctly, otherwise $\tilde{P}_{i}=0, h_{r_{i}, d}$ is the estimated channel coefficient between the $i$-th relay and destination, and $\eta_{r_{i}, d}$ is a zero-mean AWGN with unit variance. The transmission powers, $P_{i}, i=0,1, \cdots, N$, are allocated subject to a total power constraint of $P_{0}+\sum_{i=1}^{N} P_{i}=P[5]$. This power constraint is imposed to guarantee a fair comparison with the direct transmission scenario.

Flat Rayleigh fading channels are considered. Let $h_{u, v}$ be a generic channel coefficient representing the channel between any two nodes, where $h_{u, v}$ is modeled as zero-mean complex Gaussian random variable with variance $\delta_{u, v}^{2}$. The channel gain squared $\left|h_{u, v}\right|^{2}$ follows an exponential random variable with mean $\delta_{u, v}^{2}[18]$. We assume that the channel coefficients between each two nodes are independent of each other $[4,5]$, which can be practically achieved by deploying the nodes far enough from each other.

Below, we illustrate the performance degradation due to channel estimation error in the direct transmission case. For the direct transmission scenario defined in (2), the destination applies the conventional matched filter [18] as $h_{s, d}^{*} y_{s, d}$. The output SNR, denoted as $\gamma$, can be computed as

$$
\gamma^{D}=\frac{P}{N_{0}+\alpha P}\left|h_{s, d}\right|^{2}
$$

In the perfect channel estimation scenario, i.e., $\alpha=0$, the SNR at the destination increases with the data transmission power $P$. However with channel estimation error, increasing the data transmission power cannot lead to arbitrarily large SNR. This limits the performance of the direct transmission scenario and causes dramatic performance degradation. We also note that the effect of the channel 
estimation error, which is $\alpha P$ in (5), increases with high data transmission power. Motivated by the bad impact of channel estimation error on the direct transmission scenario, we investigate in the next section the ability of the various cooperative transmission protocols to mitigate such impact.

\section{Effects of Cooperative Communications}

\subsection{On Channel Estimation Error}

In this subsection, we analyze the performance of the direct and cooperative transmission scenarios introduced in Section 2. For each scenario, we calculate the outage probability and the SNR gap ratio, which is defined as

$$
R=\frac{\left.\gamma\right|_{(\alpha=0)}-\left.\gamma\right|_{(\alpha \neq 0)}}{E\left\{\left.\gamma\right|_{(\alpha=0)}\right\}},
$$

where $E\{$.$\} denotes the statistical expectation of a particular random variable. Intuitively, the SNR$ gap ratio measures the reduction in the SNR, $\left(\left.\gamma\right|_{(\alpha=0)}-\left.\gamma\right|_{(\alpha \neq 0)}\right)$, compared to the average SNR without channel estimation error, i.e., it measures the relative SNR gap ratio.

For the direct transmission scenario defined in (2), the output SNR in (5) is an exponential random variable with mean $\left(P \delta_{s, d}^{2}\right) /\left(N_{0}+\alpha P\right)$, i.e., $\gamma^{D} \sim \exp \left(\left(N_{0}+\alpha P\right) /\left(P \delta_{s, d}^{2}\right)\right)$. The outage probability, which is defined as the probability that the output SNR is less than a particular threshold $\gamma_{t h}$, is computed as

$$
F_{\gamma^{D}}\left(\gamma_{t h}\right) \triangleq \operatorname{Pr}\left(\gamma^{D} \leq \gamma_{t h}\right)=1-\exp \left(-\frac{N_{0}+\alpha P}{P \delta_{s, d}^{2}} \gamma_{t h}\right)
$$

By substituting (5) into (6), the direct transmission SNR gap ratio can be written as

$$
R^{D}=\frac{\alpha P}{\delta_{s, d}^{2}\left(N_{0}+\alpha P\right)}\left|h_{s, d}\right|^{2}
$$

The source-destination channel gain squared $\left|h_{s, d}\right|^{2}$ is an exponential random variable with mean $\delta_{s, d}^{2}$. Hence, the direct transmission SNR gap ratio in (8) is an exponential random variable, i.e., $R^{D} \sim \exp \left(\left(N_{0}+\alpha P\right) /(\alpha P)\right)$. Finally, the average SNR gap ratio can be calculated as

$$
E\left\{R^{D}\right\}=\frac{\alpha P}{N_{0}+\alpha P}
$$


In the conventional cooperative transmission scenario, the destination applies maximal-ratio combining (MRC) [19] to coherently combine the signals received from the source and the $N$ relays. The output of the MRC detector at the destination is given by

$$
y^{C}=\frac{\sqrt{P_{0}}}{N_{0}+\alpha P_{0}} h_{s, d}^{*} y_{s, d}^{C}+\sum_{i=1}^{N} \frac{\sqrt{\tilde{P}_{i}}}{N_{0}+\alpha \tilde{P}_{i}} h_{r_{i}, d}^{*} y_{r_{i}, d}^{C} .
$$

Let $\tilde{\mathbf{P}} \triangleq\left[P_{0}, \tilde{P}_{1}, \cdots, \tilde{P}_{N}\right]^{T}$ denote the power distribution vector, where $T$ denotes vector transpose. For a fixed power vector $\tilde{\mathbf{P}}$, the conditional SNR can be computed as

$$
\gamma^{C}(\tilde{\mathbf{P}})=\frac{P_{0}}{N_{0}+\alpha P_{0}}\left|h_{s, d}\right|^{2}+\sum_{i=1}^{N} \frac{\tilde{P}_{i}}{N_{0}+\alpha \tilde{P}_{i}}\left|h_{r_{i}, d}\right|^{2} .
$$

In the sequel, we obtain the distribution of the power vector $\tilde{\mathbf{P}}$, which is based on the transmission between the user and the $i$-th relay, modeled in (3). Without loss of generality, we assume M-PSK modulation type. The conditional SER at the $i$-th relay, which is conditioned on the the channel coefficient $h_{s, r_{i}}$, can be written as [20]

$$
\varepsilon^{h_{s, r_{i}}}=\Psi\left(\gamma_{i}\right) \triangleq \frac{1}{\pi} \int_{0}^{(M-1) \pi / M} \exp \left(-\frac{b \gamma_{i}}{\sin ^{2} \theta}\right) d \theta
$$

where $\gamma_{i}=P_{0}\left|h_{s, r_{i}}\right|^{2} /\left(N_{0}+\alpha P_{0}\right)$ is the instantaneous SNR at the $i$-th relay and $b=\sin ^{2}(\pi / M)$. By averaging (12) with respect to the exponential random variable $\left|h_{s, r_{i}}\right|^{2}$, the SER can be given by

$$
\varepsilon=F_{1}\left(1+\frac{b P_{0} \delta_{s, r_{i}}^{2}}{\left(N_{0}+\alpha P_{0}\right) \sin ^{2} \theta}\right)
$$

where $F_{1}(x(\theta))=1 / \pi \int_{0}^{(M-1) \pi / M} 1 / x(\theta) d \theta$.

As described in Section 2, the $i$-th relay retransmits the source's symbol only if it has correctly decoded that symbol. Hence the power of the $i$-th relay, $\tilde{P}_{i}, i=1,2 \cdots, N$, is distributed as a Bernoulli random variable with success probability equal to $(1-\varepsilon)$, i.e.,

$$
\tilde{P}_{i}=\left\{\begin{array}{ccl}
P_{i} & \text { w.p. } & 1-F_{1}\left(1+\frac{b P_{0} \delta_{s, r_{i}}^{2}}{\left(N_{0}+\alpha P_{0}\right) \sin ^{2} \theta}\right) \\
0 & \text { w.p. } \quad F_{1}\left(1+\frac{b P_{0} \delta_{s, r_{i}}^{2}}{\left(N_{0}+\alpha P_{0}\right) \sin ^{2} \theta}\right)
\end{array},\right.
$$

where w.p. stands for "with probability". We note that the relays' powers $\tilde{P}_{i}, i=1,2 \cdots, N$ are independent random variables since each one depends on its own source-relay channel gain $\left|h_{s, r_{i}}\right|$, which are independent of each other as assumed in Section 2. 
By averaging the conditional SNR in (11) with respect to $\tilde{\mathbf{P}}$, the cooperative transmission SNR can be obtained as

$$
\gamma^{C}=\frac{P_{0}}{N_{0}+\alpha P_{0}}\left|h_{s, d}\right|^{2}+\sum_{i=1}^{N} \frac{P_{i}}{N_{0}+\alpha P_{i}}\left(1-F_{1}\left(1+\frac{b P_{0} \delta_{s, r_{i}}^{2}}{\left(N_{0}+\alpha P_{0}\right) \sin ^{2} \theta}\right)\right)\left|h_{r_{i}, d}\right|^{2} .
$$

Let $h_{0}=\left|h_{s, d}\right|^{2} / \delta_{s, d}^{2}$ and $h_{i}=\left|h_{r_{i}, d}\right|^{2} / \delta_{r_{i}, d}^{2}, i=1,2, \cdots, N$, where $h_{i}, i=0,1, \cdots, N$, is distributed as a zero-mean complex Gaussian random variable with unit variance. Consequently, the SNR in (15) can be rewritten as

$$
\gamma^{C}=\sum_{i=0}^{N} a_{i}\left|h_{i}\right|^{2}
$$

where $a_{0}=\frac{P_{0} \delta_{s, d}^{2}}{N_{0}+\alpha P_{0}}$ and $a_{i}=\frac{P_{i} \delta_{r_{i}, d}^{2}}{N_{0}+\alpha P_{i}}\left(1-F_{1}\left(1+\frac{b P_{0} \delta_{s, r_{i}}^{2}}{\left(N_{0}+\alpha P_{0}\right) \sin ^{2} \theta}\right)\right), i=1,2, \cdots, N$. Furthermore, the outage probability is calculated as

$$
F_{\gamma C}\left(\gamma_{t h}\right)=\sum_{i=0}^{N} b_{i}\left(1-\exp \left(-\frac{\gamma_{t h}}{a_{i}}\right)\right)
$$

where $b_{i}=\prod_{k=0, k \neq i}^{N} \frac{a_{i}}{a_{i}-a_{k}}, i=0,1, \cdots, N$.

By substituting (16) into (6), the cooperative transmission SNR gap ratio can be given by

$$
R^{C}=\frac{\left.\gamma^{C}\right|_{(\alpha=0)}-\gamma^{C}}{E\left\{\left.\gamma^{C}\right|_{(\alpha=0)}\right\}}=\sum_{i=0}^{N} c_{i}\left|h_{i}\right|^{2}
$$

where

$$
\begin{aligned}
& c_{0}=\frac{\delta_{s, d}^{2}}{S} \frac{\alpha P_{0}^{2}}{N_{0}\left(N_{0}+\alpha P_{0}\right)}, \\
& c_{i}=\frac{\delta_{r_{i}, d}^{2}}{S}\left(\frac{\alpha P_{i}^{2}}{N_{0}\left(N_{0}+\alpha P_{i}\right)}-\frac{P_{i}}{N_{0}} F_{1}\left(1+\frac{b P_{0} \delta_{s, r_{i}}^{2}}{N_{0} \sin ^{2} \theta}\right)+\frac{P_{i}}{N_{0}+\alpha P_{i}} F_{1}\left(1+\frac{b P_{0} \delta_{s, r_{i}}^{2}}{\left(N_{0}+\alpha P_{0}\right) \sin ^{2} \theta}\right)\right),
\end{aligned}
$$

in which $S=E\left\{\left.\gamma^{C}\right|_{(\alpha=0)}\right\}=\left.\sum_{i=0}^{N} a_{i}\right|_{(\alpha=0)}$. The cooperative transmission SNR gap ratio defined in (18) represents a weighted sum of a set of independent chi-square random variables [18] and its probability density function $(\mathrm{PDF})$ can be written as

$$
f_{R^{C}}(r)=\sum_{i=0}^{N} \frac{d_{i}}{c_{i}} \exp \left(-\frac{r}{c_{i}}\right) U(r)
$$

where $d_{i}=\prod_{k=0, k \neq i}^{N} \frac{c_{i}}{c_{i}-c_{k}}, i=0,1, \cdots, N$. Finally, the average of the cooperative transmission SNR gap ratio is computed as

$$
E\left\{R^{C}\right\}=\sum_{i=0}^{N} c_{i}
$$




\begin{tabular}{|l|c|}
\hline Parameter & Value \\
\hline \hline Cell radius & $1 \mathrm{~km}$ \\
\hline Site-to-site distance & $2 \mathrm{~km}$ \\
\hline Thermal noise & $-100 \mathrm{dBm}$ \\
\hline Max transmission power & $25 \mathrm{dBm}$ \\
\hline Carrier frequency & $1.9 \mathrm{GHz}$ \\
\hline Propagation model & $31.5+35 \log _{10}(\mathrm{~d}$ in $\mathrm{m}) \mathrm{dB}$ \\
\hline
\end{tabular}

Table 1: Simulation parameters of a typical cellular system.

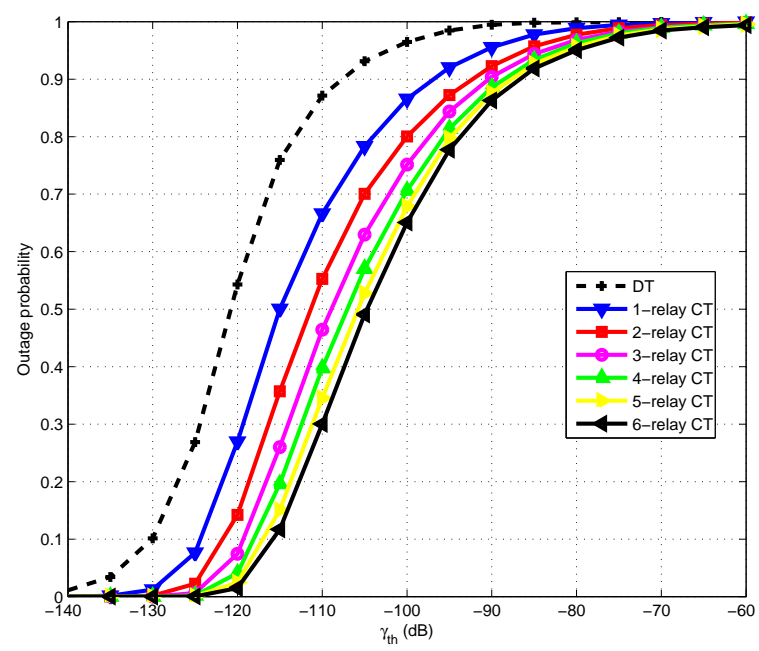

Figure 2: Channel estimator error: outage probability of the direct and cooperative transmission scenarios for $\alpha=0.05$ and $P / N_{0}=20 \mathrm{~dB}$. Cooperative transmission reduces the outage probability as the number of relays increases.

\subsubsection{Numerical Comparisons}

Now, we present some numerical results to illustrate the impact of the cooperative transmission scheme on the channel estimation error. The outage probability and SNR gap ratio are utilized to characterize such impact. For fair comparison, we assume that a total power $P$ is available for the direct and cooperative transmission scenarios. We assume maximum of $N=6$ relays are available and we consider power allocation policy, in which $P_{0}=P / 2$ and $P_{i}=P /(2 N), i=1,2, \cdots, N$ [5]. QPSK modulation type is assumed throughout this paper. Table 1 summarizes a typical set of simulation parameters for cellular networks. Finally, the shown results are averaged over 1000 independent network realizations, where the locations of the users and the relays are randomly distributed in each realization. 


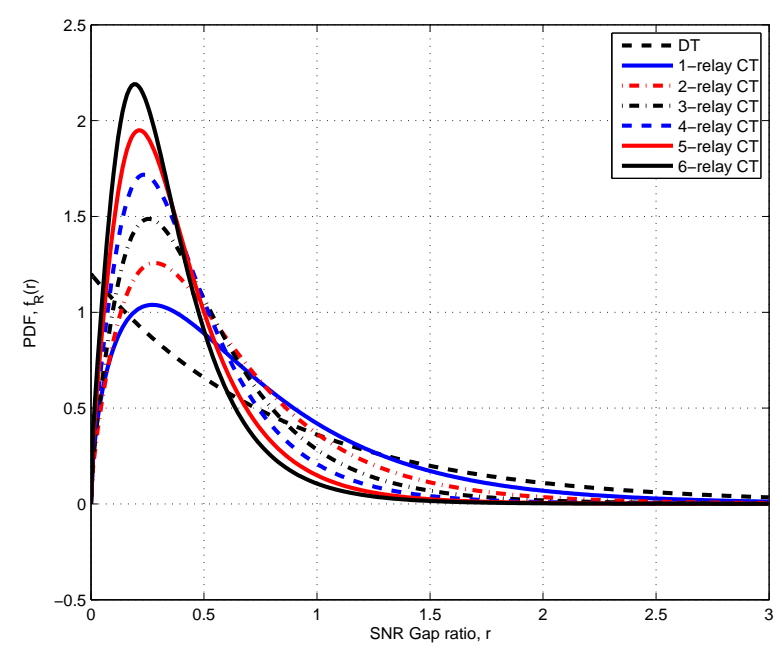

Figure 3: Channel estimator error: probability density function of the direct and cooperative transmission scenarios for $\alpha=0.05$ and $P / N_{0}=20 \mathrm{~dB}$. Direct transmission has an exponential distribution while cooperative transmission has weighted-sum chi-square distribution.

We assume that the channel estimation error variance is $\alpha=0.05$. As indicated in Section 2, the channel estimation error variance, $\alpha$, is fixed and does not depend on the data transmission power, $P$. Figure 2 depicts the outage probability, given by (7) and (17) for the direct and cooperative transmission scenarios, respectively, at $P / N_{0}=20 \mathrm{~dB}$. As shown, the direct transmission has the highest outage probability for any SNR threshold, $\gamma_{t h}$. It is also shown that as the number of relays increases, the cooperative transmission outage probability reduces. This is due to the fact that cooperative transmission with $N$ relays provides $N+1$ independently-faded paths from the source to the destination. Hence, diversity order $N+1$ is achieved.

In addition to the outage probability, the average SNR gap ratio is of great interest. Figure 3 depicts the PDF of the SNR gap ratio at $P / N_{0}=20 \mathrm{~dB}$ for the direct and cooperative transmissions. As shown, the direct transmission SNR gap ratio has an exponential distribution. On the other hand, the cooperative transmission SNR gap ratio has a weighted-sum chi-square distribution (20) that depends on the number of relays. It is also shown that as the number of relays increases, the PDF of the cooperative transmission SNR gap ratio gets more concentrated in the low-ratio region.

Figure 4 depicts the average SNR gap ratio for the different transmission scenarios as a function of $P / N_{0}$. For fixed $N_{0}$, Figure 4 shows that the average SNR gap ratio increases with the data 


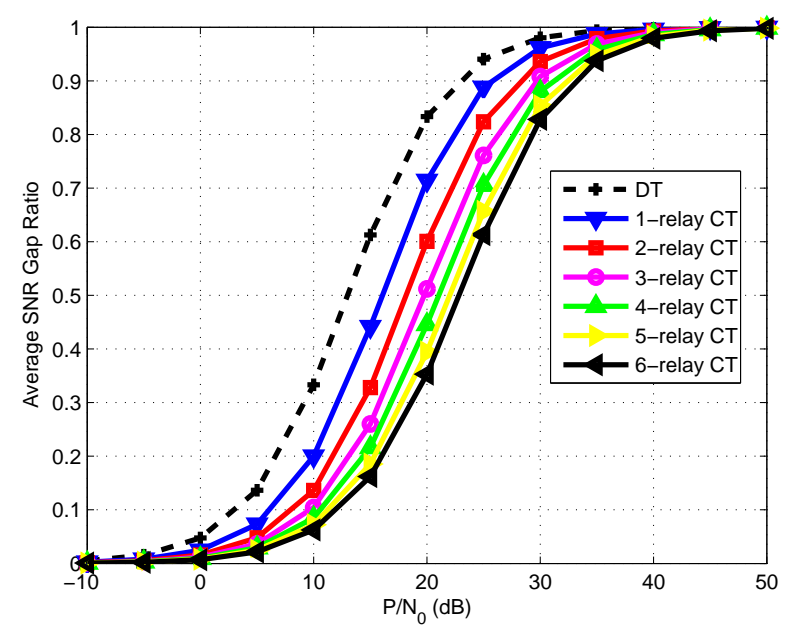

Figure 4: Channel estimator error: average SNR gap ratio of the direct and cooperative transmission scenarios for $\alpha=0.05$. Cooperative transmission reduces the average SNR gap ratio as the number of relays increases.

transmission power, $P$. This is due to the fact that the channel estimation error effect, which is $\alpha P$ in (2), is more significant at high transmission power compared to the noise variance. At high transmission power, the average SNR gap ratio is 1 as can be shown using (9). It is also depicted in Figure 4 that the direct transmission scenario has the largest SNR gap ratio compared to the conventional cooperative transmission scenario. Furthermore, increasing the number of utilized relays reduces the average SNR gap ratio. At $P / N_{0}=10 \mathrm{~dB}$, the direct transmission scenario suffers SNR gap ratio of 0.33 , while the cooperative transmission scenario with $N=6$ relays suffers SNR gap ratio of 0.06. From Figure 4, we conclude that the cooperative communication protocol reduces the effect of the channel estimation error, which is one of the main results of this paper. In this subsection, we have investigated the impact of the conventional cooperative transmission scenario on the channel estimation error. In the next subsection, we investigate such impact on the CCI problem.

\subsection{On Co-channel Interference}

In this subsection, we investigate the impact of the cooperative communications on the CCI problem in cellular networks (e.g. OFDM-based cellular networks). Reuse factor 1 is assumed, and hence the available frequency band is utilized by all the cells. For a particular mobile unit sending its data to the base station over a specific sub-carrier, it experiences a large number of interfering signals 
coming from users in its main cell as well as other cells who are occupying the same frequency sub-carrier. In each cell, there is usually a number of users transmitting their data over the same sub-carrier utilizing, for instance, space-division multiple access technique (SDMA) [21]. Moreover, a number of users can be applying MIMO schemes such as Vertical Bell Labs Space-Time Architecture (V-BLAST) [22], by which an independent symbol is transmitted from each transmit antenna over the same sub-carrier. Having reuse factor 1 in addition to these intra-cell interfering signals result in a large number of interfering signals, denoted by $K$, each contributing by a small effect. The summation of these large number of small interference quantities can be modeled, via the central limit theorem [18], as a complex Gaussian random variable. In [21], a similar argument was presented to justify approximating the inter-cell and intra-cell interference in practical systems, such as code division multiple access (CDMA) networks, as complex Gaussian random variable.

We assume that all the cells are utilizing the same transmission scenario, whether direct or cooperative transmission scenario. Below, we calculate the SINR gap ratio and the outage probability, as defined previously, for each transmission scenario. In the direct transmission scenario, the received symbol at the base station over a particular sub-carrier can be modeled as

$$
y_{s, d, C C I}^{D}=\sqrt{P} h_{s, d} x+\sum_{k=1}^{K} \sqrt{P_{k}} h_{s_{k}, d} x_{k}+\sqrt{N_{0}} \eta_{s, d},
$$

where $P_{k}$ and $x_{k}$ denote the transmission power and the unit-energy transmitted symbol of user $k$, respectively. In (22), $h_{s_{k}, d}$ represents the channel coefficient from user $k$ to the main base station and it is modeled as a zero-mean complex Gaussian random variable with variance $\delta_{s_{k}, d}^{2}$. For sufficiently large number of interferers $K$, which is a reasonable assumption as discussed above, the interference term $\left(\sum_{k=1}^{K} \sqrt{P_{k}} h_{s_{k}, d} x_{k}\right)$ can be modeled as a zero-mean complex Gaussian random variable with variance $\left(\sum_{k=1}^{K} P_{k} \delta_{s_{k}, d}^{2}\right)$. Thus, $(22)$ can be rewritten as

$$
y_{s, d, C C I}^{D}=\sqrt{P} h_{s, d} x+\sqrt{\sum_{k=1}^{K} P_{k} \delta_{s_{k}, d}^{2}+N_{0}} \eta_{s, d} .
$$

By applying matched filter at the receiver, the SINR at the main base station is given by

$$
\gamma_{C C I}^{D}=\frac{P}{N_{0}+\sum_{k=1}^{K} P_{k} \delta_{s_{k}, d}^{2}}\left|h_{s, d}\right|^{2} .
$$


As shown in (24), we cannot get arbitrarily large SINR for high transmission power $P$, and this shows the dramatic effect of the CCI on the direct transmission scenario. The outage probability, defined in (7), can be computed as

$$
F_{\gamma^{D}, C C I}\left(\gamma_{t h}\right)=1-\exp \left(-\frac{N_{0}+\sum_{k=1}^{K} P_{k} \delta_{s_{k}, d}^{2}}{P \delta_{s, d}^{2}} \gamma_{t h}\right) .
$$

Similar to (6), the SINR gap ratio due to CCI can be defined as

$$
R_{C C I}=\frac{\left.\gamma\right|_{(K=0)}-\left.\gamma\right|_{(K \neq 0)}}{E\left\{\left.\gamma\right|_{(K=0)}\right\}}
$$

Substituting (24) into (26), the direct transmission SINR gap ratio is

$$
R_{C C I}^{D}=\frac{\sum_{k=1}^{K} P_{k} \delta_{s_{k}, d}^{2}}{\left(N_{0}+\sum_{k=1}^{K} P_{k} \delta_{s_{k}, d}^{2}\right) \delta_{s, d}^{2}}\left|h_{s, d}\right|^{2},
$$

where $R_{C C I}^{D} \sim \exp \left(\left(N_{0}+\sum_{k=1}^{K} P_{k} \delta_{s_{k}, d}^{2}\right) /\left(\sum_{k=1}^{K} P_{k} \delta_{s_{k}, d}^{2}\right)\right)$. Finally, the average SINR gap ratio can be calculated as

$$
E\left\{R_{C C I}^{D}\right\}=\frac{\sum_{k=1}^{K} P_{k} \delta_{s_{k}, d}^{2}}{N_{0}+\sum_{k=1}^{K} P_{k} \delta_{s_{k}, d}^{2}} .
$$

As for the cooperative transmission mode, we assume that there exists the same number of relays in every cell. Particularly in the main cell, a set of $N$ relays help the intended user. In the $k$-th interfering cell, $k=1,2, \cdots, K$, there exists a different set of $N$ relays that are dedicated to help the $k$-th interfering user in its transmission in the $k$-th cell. In the main cell, the $N$ relays help the intended user according to their probability of correctly detecting the intended user information. On the other hand for each of the $K$ interfering cells, we assume that all the $N$ interfering relays help the corresponding interfering user. We note that this scenario represents the worst-case performance from the intended user viewpoint, since all interfering relays are always transmitting their signals and causing interference to the main cell.

In the cooperative transmission mode, the transmission scenario can be written in a similar way to that in (3) and (4) taking into consideration the CCI effect as in (23). Similar to (15), it can be shown that the received SINR is given by

$$
\begin{aligned}
\gamma_{C C I}^{C} & =\frac{P_{0}}{N_{0}+\sum_{k=1}^{K} P_{k, 0} \delta_{s_{k}, d}^{2}}\left|h_{s, d}\right|^{2} \\
& +\sum_{i=1}^{N} \frac{P_{i}}{N_{0}+\sum_{k=1}^{K} P_{k, i} \delta_{r_{k, i}, d}^{2}}\left(1-F_{1}\left(1+\frac{b P_{0} \delta_{s, r_{i}}^{2}}{\left(N_{0}+\sum_{k=1}^{K} P_{k, 0} \delta_{s_{k}, r_{i}}^{2}\right) \sin ^{2} \theta}\right)\right)\left|h_{r_{i}, d}\right|^{2},
\end{aligned}
$$




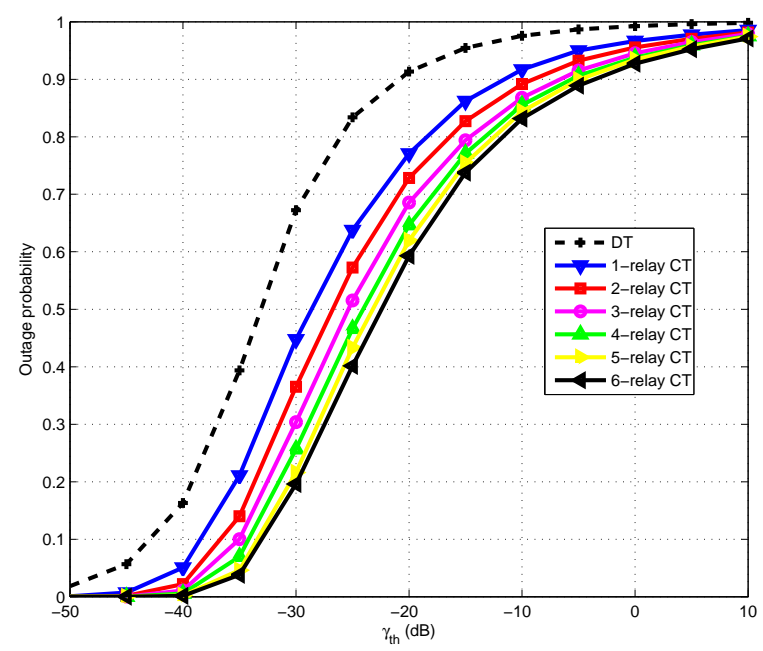

Figure 5: CCI: outage probability of the direct and cooperative transmission scenarios for equal power and $P / N_{0}=100 \mathrm{~dB}$. Cooperative transmission reduces the outage probability as the number of relays increases.

where $r_{k, i}$ denotes the $i$-th relay in the $k$-th cell and $P_{k, i}$ denotes its transmission power. The SINR in $(29)$ can be written as in (16), in which

$$
\begin{aligned}
a_{0, C C I} & =\frac{P_{0} \delta_{s, d}^{2}}{N_{0}+\sum_{k=1}^{K} P_{k, 0} \delta_{s_{k}, d}^{2}} \\
a_{i, C C I} & =\frac{P_{i} \delta_{r_{i}, d}^{2}}{N_{0}+\sum_{k=1}^{K} P_{k, i} \delta_{r_{k, i}, d}^{2}}\left(1-F_{1}\left(1+\frac{b P_{0} \delta_{s, r_{i}}^{2}}{\left(N_{0}+\sum_{k=1}^{K} P_{k, 0} \delta_{s_{k}, r_{i}}^{2}\right) \sin ^{2} \theta}\right)\right) .
\end{aligned}
$$

Similarly, the outage probability can be computed using (17), and the cooperative transmission SINR gap ratio can be given by (18), in which

$$
\begin{aligned}
c_{0, C C I} & =\frac{\delta_{s, d}^{2}}{S_{C C I}} \frac{P_{0}\left(\sum_{k=1}^{K} P_{k, 0} \delta_{s_{k}, d}^{2}\right)}{N_{0}\left(N_{0}+\sum_{k=1}^{K} P_{k, 0} \delta_{s_{k}, d}^{2}\right)}, \\
c_{i, C C I} & =\frac{\delta_{r_{i}, d}^{2}}{S_{C C I}}\left(\frac{P_{i}\left(\sum_{k=1}^{K} P_{k, i} \delta_{r_{k, i}, d}^{2}\right)}{N_{0}\left(N_{0}+\sum_{k=1}^{K} P_{k, i} \delta_{r_{k, i}, d}^{2}\right)}-\frac{P_{i}}{N_{0}} F_{1}\left(1+\frac{b P_{0} \delta_{s, r_{i}}^{2}}{N_{0} \sin ^{2} \theta}\right)+\frac{P_{i}}{N_{0}+\sum_{k=1}^{K} P_{k, i} \delta_{r_{k, i}, d}^{2}}\right. \\
& \left.\times F_{1}\left(1+\frac{b P_{0} \delta_{s, r_{i}}^{2}}{\left(N_{0}+\sum_{k=1}^{K} P_{k, 0} \delta_{s_{k}, r_{i}}^{2}\right) \sin ^{2} \theta}\right)\right),
\end{aligned}
$$

in which $S_{C C I}=E\left\{\left.\gamma_{C C I}^{C}\right|_{(K=0)}\right\}=\left.\sum_{i=0}^{N} a_{i, C C I}\right|_{(K=0)}$. Finally, the PDF and average of the cooperative transmission SINR gap ratio can be calculated as in (20) and (21), respectively.

In the sequel, we present the CCI numerical results. We assume $K=7$ interfering users, which exist in $K$ neighboring cells that have noticeable effect on the main user. In addition, we assume that 


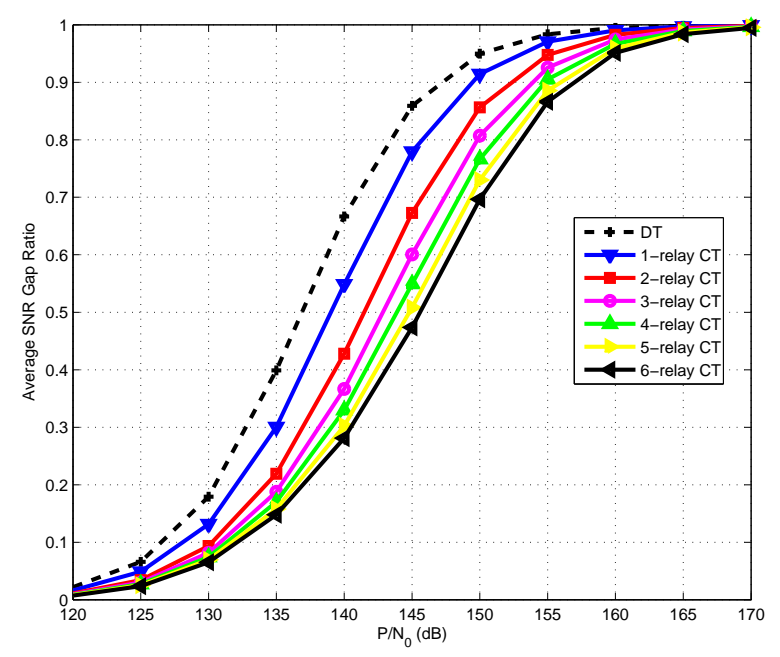

Figure 6: CCI: average SINR gap ratio of the direct and cooperative transmission scenarios for equal power. Cooperative transmission reduces the SINR gap ratio as the number of relays increases.

all the users in the various cells are having the same power allocation policy, i.e., $P_{0}=P_{k, 0}=P / 2$ and $P_{i}=P_{k, i}=P /(2 N), i=1,2, \cdots, N$. The rest of the simulation parameters are given in Table 1 . In Figure 5, we show the outage probability of the SINR for the direct and cooperative transmission scenarios. Similar to the channel estimation error case, it is shown that the cooperative transmission reduces the outage probability as the number of relays increases. Figure 6 depicts the average SNR gap ratio and it is shown that the CCI effect is reduced by utilizing relays. Moreover, increasing the number of cooperating relays results in lower SNR gap ratio. From Figure 6, we conclude that the conventional cooperative communication protocol is less susceptible to CCI compared to the direct transmission, which is one of the main results of this paper.

In this subsection, we have presented the CCI problem in a similar fashion to that of the channel estimation error. In the rest of this paper, we will focus on the channel estimation error, however, the obtained results can be easily extended to the CCI case. In the following subsections, we study the impact of additional transmission protocols, namely, relay selection and multi-phase direct transmission, on the channel estimation error effect. 


\subsection{Relay Selection}

In this subsection, we consider a different cooperative transmission scenario, namely, cooperative communications with relay selection [6]. In the relay-selection cooperative scheme, one optimal relay among a set of $N$ available relays is chosen based on the instantaneous channel gains. This protocol guarantees full diversity order of $N+1$ as was proven in [6]. Unlike the conventional cooperative scheme with bandwidth efficiency of $1 /(N+1)$ SPCU, the relay selection scheme achieves bandwidth efficiency of $1 / 2 \mathrm{SPCU}$. In [6], it was shown that the effective channel from the source to the destination via the $i$-th relay can be quantified using the following relay metric

$$
\beta_{i}=\mu_{H}\left(\frac{A^{2}}{q^{2}}\left|h_{r_{i}, d}\right|^{2}, \frac{B}{q(1-q)}\left|h_{s, r_{i}}\right|^{2}\right) \triangleq \frac{2 \frac{A^{2}}{q^{2}} \frac{B}{q(1-q)}\left|h_{s, r_{i}}\right|^{2}\left|h_{r_{i}, d}\right|^{2}}{\frac{A^{2}}{q^{2}}\left|h_{r_{i}, d}\right|^{2}+\frac{B}{q(1-q)}\left|h_{s, r_{i}}\right|^{2}},
$$

where $\mu_{H}(.,$.$) denotes the standard harmonic mean function, q \triangleq \frac{P_{o}}{P}$ represents the portion of the total transmission power assigned to the user, and for M-PSK modulation $A=\frac{M-1}{2 M}+\frac{\sin \left(\frac{2 \pi}{M}\right)}{4 \pi}$ and $B=\frac{3(M-1)}{8 M}+\frac{\sin \left(\frac{2 \pi}{M}\right)}{4 \pi}-\frac{\sin \left(\frac{4 \pi}{M}\right)}{32 \pi}[6]$.

The $i$-th relay metric $\beta_{i}$ in (32) gives an instantaneous indication about the relay's ability to cooperate with the user. Consequently, the optimal relay is the one that has the maximum instantaneous relay metric among the set of available relays. The user utilizes the optimal relay only to forward its data to the destination. The relay-selection cooperative scheme can be modeled by (3) and (4) utilizing one relay only, i.e., $K=1$. Let $\tilde{P}_{m}$ denote the transmission power of the optimal relay, $r_{m}$. Similar to (11), for a given $\tilde{P}_{m}$ the conditional SNR is calculated as

$$
\gamma^{S}\left(\tilde{P_{m}}\right)=\frac{P_{0}}{N_{0}+\alpha P_{0}}\left|h_{s, d}\right|^{2}+\frac{\tilde{P}_{m}}{N_{0}+\alpha \tilde{P_{m}}}\left|h_{r_{m}, d}\right|^{2},
$$

where the superscript $\mathrm{S}$ denotes relay selection scheme. The transmission power of the optimal relay $P_{m}$ is a Bernoulli random variable, with PDF given by (14). By averaging (33) with respect to $P_{m}$, the SNR can be computed as

$$
\gamma^{S}=\frac{P_{0}}{N_{0}+\alpha P_{0}}\left|h_{s, d}\right|^{2}+\frac{P_{m}}{N_{0}+\alpha P_{m}}\left(1-F_{1}\left(\exp \left(\frac{b P_{0}\left|h_{s, r_{m}}\right|^{2}}{\left(N_{0}+\alpha P_{0}\right) \sin ^{2} \theta}\right)\right)\right)\left|h_{r_{m}, d}\right|^{2} .
$$

We note that the channel gains of the optimal relay, namely, $\left|h_{s, r_{m}}\right|^{2}$ and $\left|h_{r_{m}, d}\right|^{2}$ are no longer exponentially distributed random variables as was shown in [6]. It is very complicated to analytically 


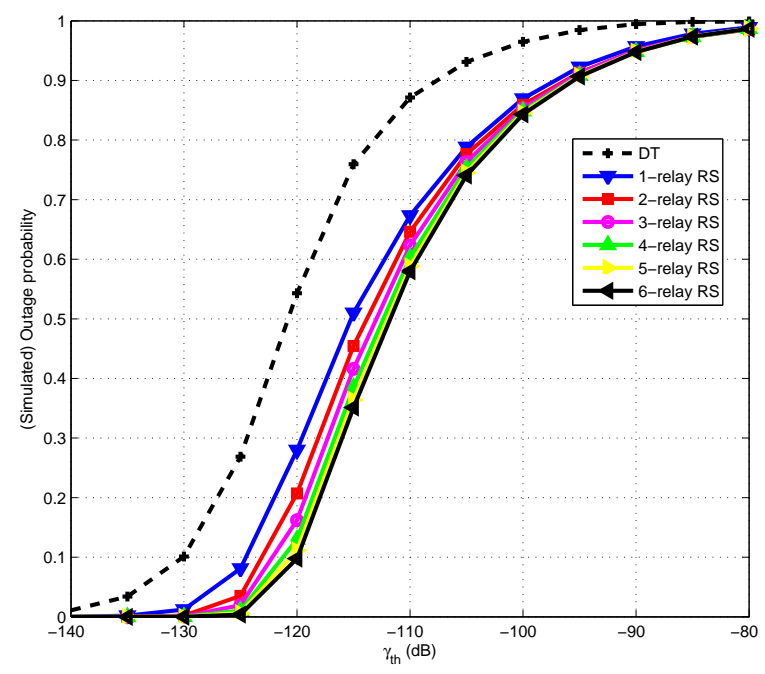

Figure 7: Channel estimator error: outage probability of the direct and relay-selection cooperative transmission scenarios for $P / N_{0}=20 \mathrm{~dB}$ and $\alpha=0.05$. Cooperative transmission reduces the outage probability as the number of relays increases.

obtain the probability distribution of the optimal relay channels. Therefore, we show by simulations the performance of the relay-selection cooperative scheme.

The simulated outage probability of the relay-selection cooperative transmission scheme at $P / N_{0}=$ $20 \mathrm{~dB}$ and $\alpha=0.05$ is depicted in Figure 7. As shown, the outage probability of the cooperative protocol is lower than that of the the direct transmission and it decreases as the number of relays increases due to achieving diversity order equal to $N+1$. Figure 8 depicts the average SNR gap ratio of the relay-selection cooperative transmission scenario. As shown, all the relay-selection curves with different number of available relays have the same average SNR gap ratio, which is lower than that of the direct transmission scenario. Moreover, the average SNR gap ratio of the relay-selection scheme is the same as that of the conventional cooperative scheme with one relay only, which was shown in Figure 4. From Figure 8, we conclude that relay-selection cooperative scheme does not reduce the effect of the channel estimation error by adding more relays. This case is different from the conventional cooperative scheme, in which increasing the number of relays reduces the effect of the channel estimation error. From Figure 4 and Figure 8, we conclude that achieving higher diversity order is not the only factor for mitigating the effect of channel estimation error. In order to find out 


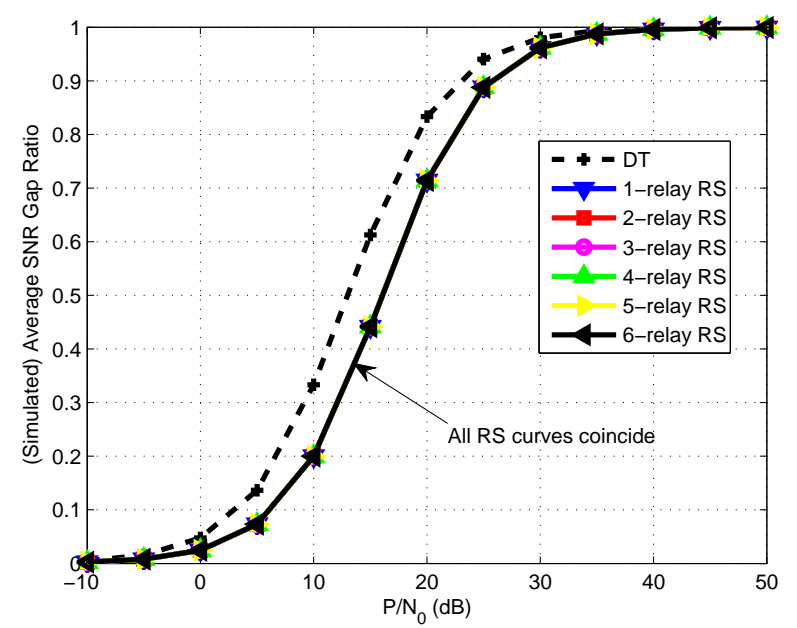

Figure 8: Channel estimator error: average SNR gap ratio of the direct and relay-selection cooperative transmission scenarios for $\alpha=0.05$. The average SNR gap ratio is almost constant as the number of relays increases.

the other factors, we consider in the following subsection the multi-phase direct transmission scheme.

\subsection{Multi-phase Direct transmission}

In this subsection, we consider the multi-phase direct transmission scenario, in which a user sends its data to its destination in $N$ consecutive channel uses, each with a transmission power of $P / N$. There is no relays utilized in this scheme. Similar to (8), it can be shown that the SNR gap ratio is given by

$$
R^{D}(N)=\frac{\alpha P}{N \delta_{s, d}^{2}\left(N_{0}+\alpha P / N\right)}\left|h_{s, d}\right|^{2},
$$

i.e., $R^{D}(N) \sim \exp \left(N\left(N_{0}+\alpha P / N\right) /(\alpha P)\right)$. The average SNR gap ratio can be calculated as

$$
E\left\{R^{D}(N)\right\}=\frac{\alpha P}{N\left(N_{0}+\alpha P / N\right)}
$$

We note that the multi-phase direct transmission scenario achieves diversity order equal to 1 , and has outage probability similar to that of the conventional direct transmission. Figure 9 depicts the average SNR gap ratio for the multi-phase and conventional cooperative transmission scenarios. As shown, the multi-phase direct transmission protocol reduces the SNR gap ratio as the number of relays increases. Therefore by distributing the total transmission power across multiple transmission phases, the effect of the channel estimation error can be mitigated. In Figure 9, it is also shown that 


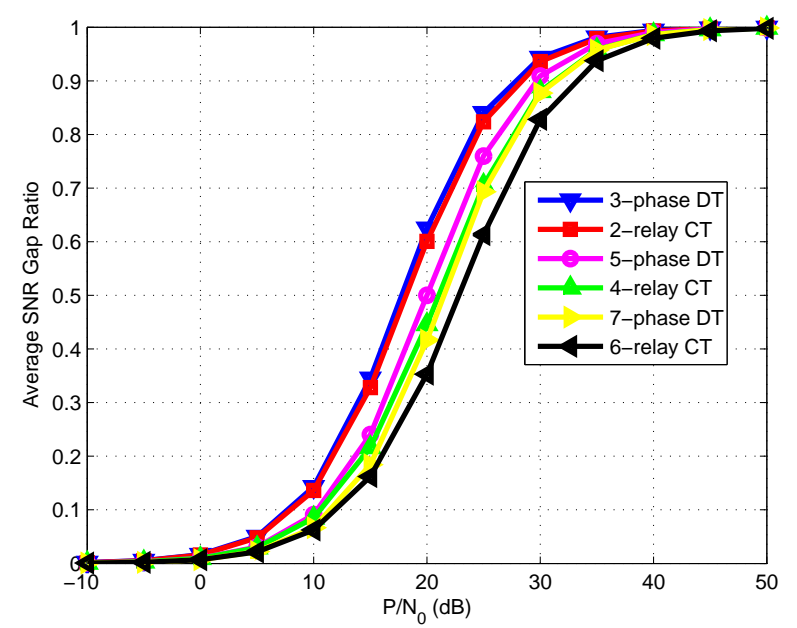

Figure 9: Channel estimator error: average SNR gap ratio of the multi-phase direct and cooperative transmission scenarios for $\alpha=0.05$. Cooperative transmission scenarios reduces the SNR gap ratio more than the multi-phase direct transmission for the same number of phases.

the cooperative transmission scenario reduces the SNR gap ratio more compared to the multi-phase direct transmission, for the same total number of transmission phases.

From Figure 4, Figure 8, and Figure 9, we conclude that the reduction in the SNR gap ratio is due to two main factors. The first factor is the distribution of the transmission power across multiple transmission phases. This reduces the transmission power in each phase, and accordingly the channel estimation error portion, $\alpha P$, in each transmission is reduced. This first reduction factor exists in both the multi-phase direct transmission and conventional cooperative transmission scenarios, and hence both of them mitigate the effect of channel estimation error by increasing the number of transmission phases as was shown in Figure 9. On the contrary, the relay-selection cooperative scheme does not distribute the transmission power more by having more available relays.

The second factor of reducing the effect of channel estimation error is the achieved diversity order. The conventional cooperative transmission scenario utilizes relays, other than retransmission over the same channel. The cooperation gain resulting from utilizing relays reduces the channel estimation error effect more. This is clear in the SNR gap ratio PDF, as was shown previously in Figure 3, where the direct transmission SNR gap ratio is exponentially distributed while the conventional cooperative transmission SNR gap ratio is distributed as a weighted sum chi-square random variable. Since the 


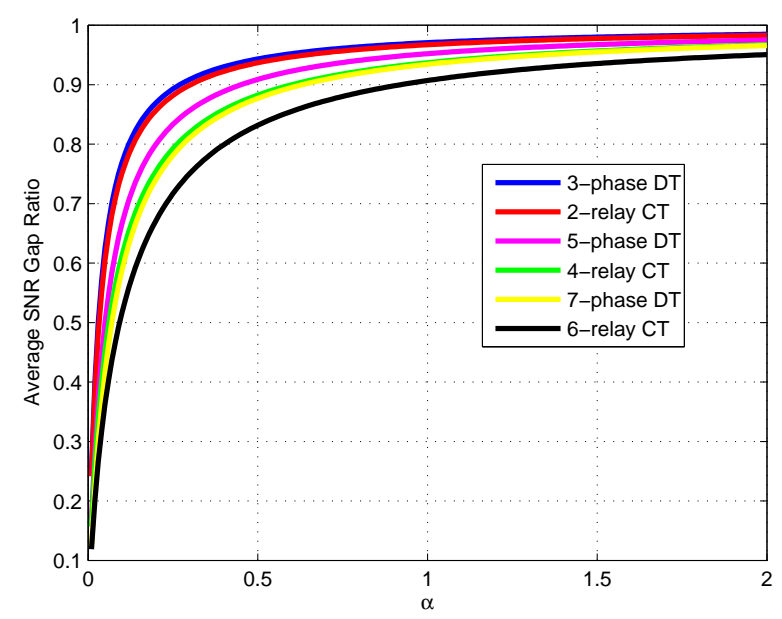

Figure 10: Channel estimator error: average SNR gap ratio of the multi-phase direct and cooperative transmission scenarios for $P / N_{0}=20 \mathrm{~dB}$. Cooperative transmission scenarios reduces the SNR gap ratio more than the multi-phase direct transmission for the same number of phases.

conventional cooperative scheme achieves full diversity order along with distributing the transmission power, it reduces the SNR gap ratio compared to that of the multi-phase direct transmission, as was shown in Figure 9.

Finally, Figure 10 depicts the effect of the channel estimation error variance $\alpha$ on the average SNR gap ratio at $P / N_{0}=20 \mathrm{~dB}$. As expected, the average SNR gap ratio increases as $\alpha$ increases. Moreover, it is shown that cooperative transmission reduces the SNR gap ratio compared to the multi-phase direct transmission for the same number of transmission phases, which agrees with the result previously shown in Figure 9.

\section{Timing Synchronization Error}

In addition to the conventional and relay-selection cooperative schemes, we consider distributed space-time cooperative schemes $[7,8,16]$, in which all the cooperating relays are simultaneously transmitting their designated codes. Assuming perfect timing synchronization among the relays, distributed space-time cooperative schemes achieve bandwidth efficiency equal to $1 / 2$ SPCU while guaranteeing full diversity order [8]. Distributed space-time cooperative schemes suffer from timing synchronization error, which is a result of having the start of the transmission time of the cooperat- 
ing relays not completely synchronized with each other. Moreover, due to the different geographic locations of the relays, signals transmitted from different relays experience different propagation delays and consequently arrive at the destination at different time instants. The destination picks a particular sampling instant, which definitely does not match the signals from all the relays. At the chosen sampling instant, the destination reads the mixture of a number of interfering signals that come from various multipaths, which dramatically increases the error rate. Finally, we note that the timing synchronization error increases as the number of relays increases.

In distributed space-time cooperative communication, there are two main contradicting factors that affect the system performance, which are the channel estimation error and the timing synchronization error. Channel estimation error effect decreases as the number of relays increases, as was previously shown in Figure 4. On the contrary, timing synchronization error increases as the number of relays increases. In this section, we investigate the tradeoff between these two contradicting types of error and their net impact on the system performance. In particular, we analyze one of the distributed space-time cooperative schemes, namely, distributed transmit beamforming scheme [16]. In distributed transmit beamforming transmission, the set of cooperating relays applies transmit beamforming via the available instantaneous relay-destination channel gain at each relay.

The distributed transmit beamforming scheme can be implemented in two consecutive transmission phases as follows. In the first phase, the source broadcasts its symbol, which is received by the set of $N$ relays and the destination. The received symbols at the destination and the $i$-th relay can be modeled as in (3), with $P_{0}=P / 2$. Each relay decodes the received symbol and transmits it to the destination if correctly decoded, otherwise, it remains idle. The $k$-th transmitted sample from the $i$-th relay at time $k T$, where $T$ is the symbol time, is given by

$$
x_{i}(k)=I_{r_{i}}(k) \frac{h_{r_{i}, d}(k)^{*}}{\left\|\mathbf{h}_{r, d}(k)\right\|} x(k),
$$

where $\mathbf{h}_{r, d}(k)=\left[h_{r_{1}, d}(k), h_{r_{2}, d}(k), \cdots, h_{r_{N}, d}(k)\right]^{T}$ and $\|$.$\| denotes the vector norm. In (37), I($.$) is$ the indicator function of the $i$-th relay and it is given by

$$
I_{r_{i}}(k)=\left\{\begin{array}{ll}
1, & \text { if } r_{i} \text { correctly decoded the } k \text {-th symbol } \\
0, & \text { Otherwise }
\end{array},\right.
$$


Each relay multiples its transmitted sample by a pulse shape function, denoted by $p($.$) , before trans-$ mission. We consider raised cosine pulse shape, $p($.$) , with roll-off factor of 0.5$. In this paper, we take into consideration the contribution from the first-order sidelobes of $p($.$) and neglect that of the$ higher-order sidelobes due to its smaller effect [23]. We assume that the sampling instant at the destination is $\left(k T+\Delta_{o}\right)$, where $\Delta_{o}$ is a timing shift chosen by the destination to compensate for the average propagation delay. The received signal at the destination can be written as

$$
y^{B}(k)=\sqrt{P / 2} \sum_{i=1}^{N} \sum_{l=-1}^{1} x_{i}(k-l)\left(h_{r_{i}, d}(k-l)+h_{r_{i}, \alpha}(k-l)\right) p\left(\Delta_{o}-T_{i}+l T\right)+\eta(k),
$$

where the superscript B denotes the distributed beamforming scheme. In (39), $T_{i}$ is the propagation delay of the $i$-th relay and $h_{r_{i}, \alpha}$ represents the channel estimation error at the destination for the channel from the $i$-th relay to the destination. Finally, $\eta(k)$ is a zero-mean AWGN with variance $N_{o}$.

The received signal in (39) can be rewritten as

$$
y^{B}(k)=y_{\text {des }}(k)+y_{\text {int }}(k)+\eta_{\alpha}(k),
$$

where $y_{\text {des }}(),. y_{\text {int }}($.$) , and \eta_{\alpha}($.$) denote the desired signal, the interference signal, and the noise term$ including the channel estimation error, respectively. The desired signal is given by

$$
y_{\text {des }}(k)=\sqrt{P / 2} \frac{x(k)}{\|\mathbf{h}(k)\|} \sum_{i=1}^{N}\left(\left|h_{r_{i}, d}(k)\right|^{2} I_{r_{i}}(k) p\left(\Delta_{o}-T_{i}\right)\right) .
$$

In addition, the interference signal can be modeled as

$$
\begin{aligned}
y_{\text {int }}(k) & =\sqrt{P / 2} \frac{x(k+1)}{\|\mathbf{h}(k+1)\|} \sum_{i=1}^{N}\left(\left|h_{r_{i}, d}(k+1)\right|^{2} I_{r_{i}}(k+1) p\left(\Delta_{o}-T_{i}-T\right)\right) \\
& +\sqrt{P / 2} \frac{x(k-1)}{\|\mathbf{h}(k-1)\|} \sum_{i=1}^{N}\left(\left|h_{r_{i}, d}(k-1)\right|^{2} I_{r_{i}}(k-1) p\left(\Delta_{o}-T_{i}+T\right)\right) .
\end{aligned}
$$

The composite noise term is

$$
\eta_{\alpha}(k)=\sqrt{P / 2} \sum_{i=1}^{N} \sum_{l=-1}^{1} \frac{x(k-l)}{\|\mathbf{h}(k-l)\|}\left(h_{r_{i}, d}(k-l)^{*} h_{r_{i}, \alpha}(k-l) I_{r_{i}}(k-l) p\left(\Delta_{o}-T_{i}+l T\right)\right)+\eta(k) .
$$

The channel estimation error terms, $h_{r_{i}, \alpha}$, for different relays $r_{i}$ and time instants $l T$ are independent and identically distributed with variance $\alpha$. For a fixed $x$ and $\mathbf{h}$, the noise variance can be calculated as

$$
E\left\{\eta_{\alpha}(k)^{2}\right\}=\frac{\alpha P}{2} \sum_{i=1}^{N} \sum_{l=-1}^{1}\left(\frac{\left|h_{r_{i}, d}(k-l)\right|^{2}}{\|\mathbf{h}(k-l)\|^{2}} I_{r_{i}}(k-l) p^{2}\left(\Delta_{o}-T_{i}+l T\right)\right)+N_{o} .
$$




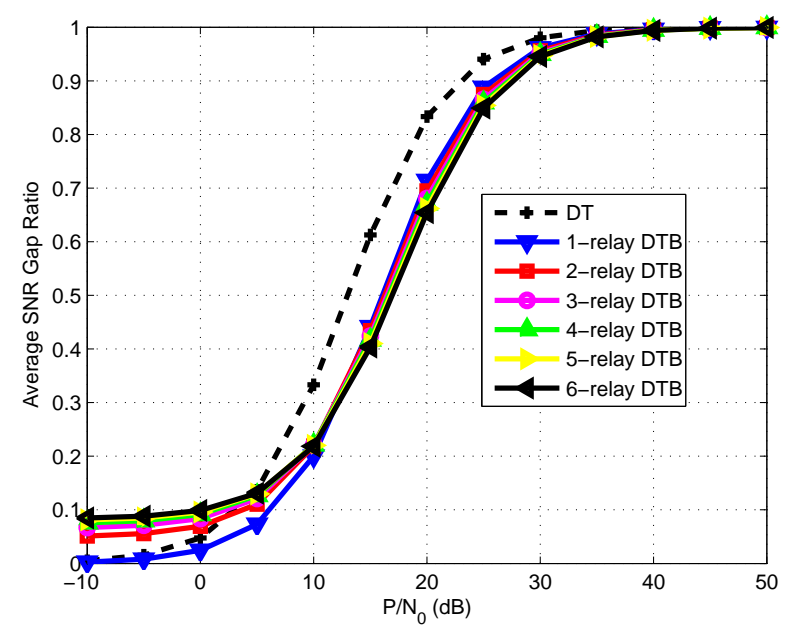

Figure 11: Channel estimator error: average SINR gap ratio of the direct and distributed transmit beamforming cooperative transmission scenarios for $\alpha=0.05$ and $\Delta T=0.15 T$. The average SNR gap ratio increases at low transmission power, and decreases at high transmission power with increasing the number of relays.

Finally, the conditional received SINR can be computed as

$$
\gamma^{B}(k)=\frac{P}{2\left(N_{0}+\alpha P / 2\right)}\left|h_{s, d}(k)\right|^{2}+\frac{y_{\text {des }}(k)^{2}}{y_{\text {int }}(k)^{2}+E\left\{\eta_{\alpha}(k)^{2}\right\}},
$$

where the first term represents the SNR due to the first phase, which is similar to the first term in (11).

We assume that the timing synchronization error is distributed uniformly as $\left(\Delta_{o}-T_{i}\right) \sim U[-\Delta T / 2, \Delta T / 2]$. Figure 11 depicts the average SINR gap ratio for a particular deployment scenario, in which the relays are close to the middle between the source and destination and $\Delta T=0.15 T$. The average SINR gap ratio is obtained via simulations by averaging over independent channel and independent timing synchronization error realizations. For each realization, the SINR and SINR gap ratio are calculated as in (45) and (6), respectively. In (6), $\left.\gamma\right|_{(\alpha=0)}$ refers to having perfect channel estimation and perfect timing synchronization case. Finally, the outage probability is calculated based on the SINR expression given in (45).

Figure 11 depicts the average SINR gap ratio of the distributed transmit beamforming scheme. As shown, the net impact of the two contradicting error effects depends on the data transmission power, $P$. We note that the timing synchronization error effect does not depend on the transmission 


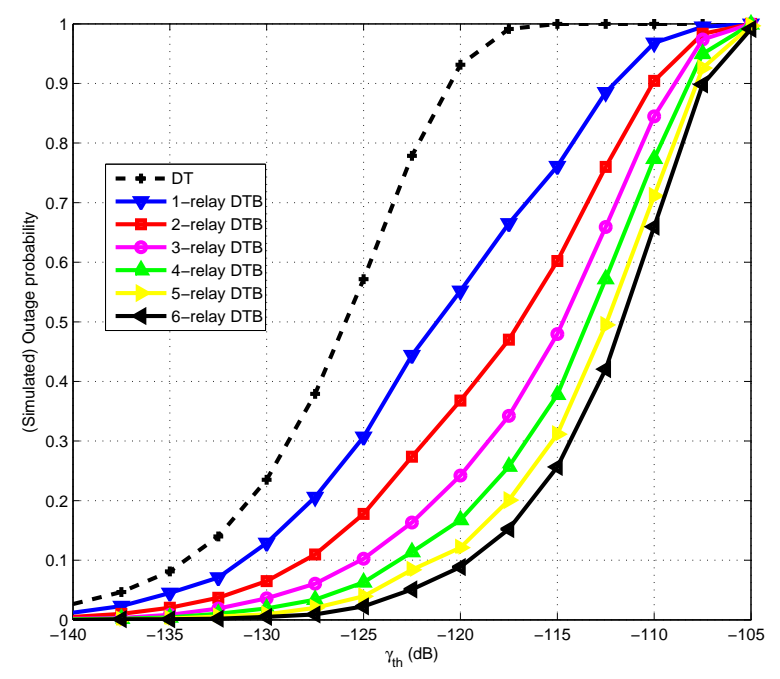

Figure 12: Channel estimator error: outage probability of the direct and distributed transmit beamforming transmission scenarios for $\alpha=0.05, \Delta T=0.15 T$, and $P / N_{0}=20 \mathrm{~dB}$. Distributed transmit beamforming transmission reduces the outage probability as the number of relays increases.

power. On the contrary, the effect of the channel estimation error, $\alpha P$, increases with increasing the data transmission power. At low transmission power, the effect of the synchronization error is more significant compared to that of the channel estimation error. Hence at low transmission power, having more relays increases the average SINR gap ratio as shown in Figure 11. As the transmission power increases, the effect of channel estimation error gets more significant compared to that of the timing synchronization error. Therefore at high transmission power, adding more relays leads to net effect of lower average SINR gap ratio. Finally, Figure 12 depicts the outage probability of the distributed transmit beamforming transmission at $P / N_{0}=20$. As shown, the outage probability decreases as the number of relays increases due to achieving higher spatial diversity order, which is equal to $N+1$.

\section{Conclusion}

In this paper, we have investigated the impact of the cooperative communications on mitigating channel estimation error and co-channel interference (CCI) effects. The SNR gap ratio, which measures the reduction in the SNR, and the conventional outage probability were utilized to characterize the 
system performance. We have shown that the cooperative transmission schemes are less susceptible to the channel estimation error compared to the direct transmission. Furthermore, increasing the number of relays results in lower SNR gap ratio. At $P / N_{0}=10 \mathrm{~dB}$ and channel estimation error variance $\alpha=0.05$, the direct transmission scenario suffers SNR gap ratio of 0.33 , while the cooperative transmission scenario with $N=6$ relays suffers SNR gap ratio of 0.06 only. We have illustrated that cooperative transmission reduces the channel estimation error effect due to two main factors: (1) achieving spatial diversity via relays and (2) distributing the transmission power across multiple transmission phases.

We have also considered distributed transmit beamforming cooperative scheme, and we have studied the tradeoff between the timing synchronization error and channel estimation error. At low data transmission power, the timing synchronization error is more significant. As the data transmission power increases, we find that the effect of channel estimation error overcomes that of the timing synchronization error. Finally, we have shown that cooperative schemes are less susceptible to the CCI problem, compared to that of the direct transmission.

\section{References}

[1] K. J. R. Liu, A. K. Sadek, W. Su, and A. Kwasinski, Cooperative communications and networking, Cambridge University Press, 2008.

[2] J. N. Laneman, D. N. C. Tse, and G. W. Wornell, "Cooperative diversity in wireless networks: efficient protocols and outage behavior," IEEE Trans. Information Theory, vol. 50, no. 12, pp. 3062-3080, Dec. 2004.

[3] P. Mitran, H. Ochiai, and V. Tarokh, "Space-time diversity enhancements using collaborative communications," IEEE Trans. on Information Theory, vol. 51, no. 6, pp. 2041 - 2057, Jun. 2005.

[4] W. Su, A. K. Sadek, and K. J. R. Liu, "Cooperative communication protocols in wireless networks: performance analysis and optimum power allocation," Wireless Personal Communications, vol. 44, pp. 181-217, Jan. 2008.

[5] A. K. Sadek, W. Su, and K. J. R. Liu, "Multi-node cooperative communications in wireless networks," IEEE Trans. Signal Processing, vol. 55, pp. 341-355, Jan. 2007.

[6] A. S. Ibrahim, A. K. Sadek, W. Su, and K. J. R. Liu, "Cooperative communications with relay-selection: when to cooperate and whom to cooperate with?," IEEE Trans. on Wireless Comm., vol. 7, pp. 2814 - 2827, Jul. 2008.

[7] J. N. Laneman and G. W. Wornell, "Distributed space-time coded protocols for exploiting cooperative diversity in wireless networks," IEEE Trans. Information Theory, vol. 49, no. 10, pp. 2415-2425, Oct. 2003.

[8] K. G. Seddik, A. K. Sadek, A. S. Ibrahim, and K. J. R. Liu, "Design criteria and performance analysis for distributed space-time coding," IEEE Trans. on Vehicular Technology, vol. 57, pp. 2280 - 2292, Jul. 2008.

[9] H. Cheon and D. Hong, "Effect of channel estimation error in OFDM-based WLAN," IEEE Communications Letters, vol. 6, pp. 190 - 192, May 2002. 
[10] L. Li, X. Zhao, H. Hu, and H. Yu, "Effect of imperfect channel estimation on multi-branch cooperative links in fading channels," Proc. IEEE International Conference on Wireless Communications, Networking and Mobile Computing(WiCom'07), pp. 1087 - 1090, Sep. 2007.

[11] H. Jin, R. Laroia, and T. Richardson, "Superposition by position," Proc. IEEE Information Theory Workshop, pp. 222 - 226, Mar. 2006.

[12] F. Rashid-Farrokhi, K. J. R. Liu, and L. Tassiulas, "Transmit beamforming and power control for cellular wireless systems," IEEE Journal of Selected Areas in Communications, Special Issue on Signal Processing for Wireless Communications, vol. 16, pp. 1437 - 1450, Oct. 1998.

[13] F. Rashid-Farrokhi, L. Tassiulas, and K. J. R. Liu, "Joint optimal power control and beamforming in wireless networks using antenna arrays," IEEE Trans. on Communications, vol. 46, pp. 1313 - 1324, Oct. 1998.

[14] W. Choi, N. Himayat, S. Talwar, and M. Ho, "The effects of co-channel interference on spatial diversity techniques," Proc. IEEE Wireless Communications and Networking Conference (WCNC'07), pp. 1938 - 1943, Mar. 2007.

[15] Y. Akyildiz and B.D. Rao, "Statistical performance analysis of optimum combining with co-channel interferers and flat rayleigh fading," Proc. IEEE Global Telecommunications Conference (GLOBECOM'01), vol. 6, pp. 3663 - 3667, Nov. 2001.

[16] S. Jagannathan, H. Aghajan, and A. Goldsmith, "The effect of time synchronization errors on the performance of cooperative MISO systems," Proc. IEEE Global Telecommunications Conference (GLOBECOM'04), pp. 102 107, Nov. 2004.

[17] T-D. Nguyen, O. Berder, and O. Sentieys, "Impact of transmission synchronization error and cooperative reception techniques on the performance of cooperative MIMO systems," Proc. IEEE International Conference on Communications (ICC '08), pp. 4601 - 4605, May 2008.

[18] J. G. Proakis, Digital Communications, McGraw-Hill Inc., 4th ed., 2000.

[19] D. G. Brennan, "Linear diversity combining techniques," Proc. IEEE, vol. 91, no. 2, pp. 331-356, Feb. 2003.

[20] M.K. Simon and M.-S. Alouini, "A unified approach to the performance analysis of digital communication over generalized fading channels," Proc. IEEE, vol. 86, pp. 1860-1877, Sep. 1998.

[21] D. Tse and P. Viswanath, Fundamentals of wireless communication, Cambridge University Press, 2005.

[22] G. J. Foschini, "Layered space-time architecture for wireless communication in a fading environment when using multiple antennas," Bell Lab. Tech. J., vol. 1, pp. 41-59, 1996.

[23] Y. Mei, Y. Hua, A. Swami, and B. Daneshrad, "Combating synchronization errors in cooperative relays," Proc. IEEE International Conference on Acoustics, Speech, and Signal Processing (ICASSP '05), pp. iii/369 - iii/372, Mar. 2005. 
Ahmed S. Ibrahim (M'09) received the B.S. (with highest honors) and M.S. degrees in electronics and electrical communications engineering from Cairo University, Cairo, Egypt, in 2002 and 2004, respectively. He received the Ph.D. degree in electrical engineering from the University of Maryland, College Park, MD, USA, in 2009.

From 2002 to 2004, he was a Teaching/Research Assistant at Cairo University. From 2004 to 2009, he was a Research Assistant at the University of Maryland. Currently, he is a Research Scientist at Chalmers University of Technology, Gothenburg, Sweden. His research interests include crosslayer design of wireless networks, cooperative communications and networking, relay deployment and selection in wireless networks, and MIMO-OFDM systems.

Dr. Ibrahim is a recipient of the best speaker award of the Electrical and Computer Engineering Department seminar series at the University of Maryland in 2008. He was a nominee for the best student paper award of the IEEE Conference on Acoustics, Speech, and Signal Processing (ICASSP) in 2007. He is also a recipient of the Graduate School Fellowship from the University of Maryland in 2004 and 2005 .

K. J. Ray Liu (F'03) is a Distinguished Scholar-Teacher of University of Maryland, College Park. He is Associate Chair of Graduate Studies and Research of Electrical and Computer Engineering Department and leads the Maryland Signals and Information Group conducting research encompassing broad aspects of information technology including communications and networking, information forensics and security, multimedia signal processing, and biomedical technology.

Dr. Liu is the recipient of numerous honors and awards including best paper awards from IEEE Signal Processing Society, IEEE Vehicular Technology Society, and EURASIP; IEEE Signal Processing Society Distinguished Lecturer, EURASIP Meritorious Service Award, and National Science Foundation Young Investigator Award. He also received various teaching and research recognitions from University of Maryland including university-level Invention of the Year Award; and Poole and Kent Senior Faculty Teaching Award and Outstanding Faculty Research Award, both from A. James Clark School of Engineering. Dr. Liu is a Fellow of IEEE and AAAS. 
Dr. Liu was Vice President - Publications and will serve as President-Elect from 2010. He was the Editor-in-Chief of IEEE Signal Processing Magazine and the founding Editor-in-Chief of EURASIP Journal on Applied Signal Processing.

His recent books include Cooperative Communications and Networking, Cambridge University Press, 2008; Resource Allocation for Wireless Networks: Basics, Techniques, and Applications, Cambridge University Press, 2008; Ultra-Wideband Communication Systems: The Multiband OFDM Approach, IEEE-Wiley, 2007; Network-Aware Security for Group Communications, Springer, 2007; Multimedia Fingerprinting Forensics for Traitor Tracing, Hindawi, 2005; Handbook on Array Processing and Sensor Networks, IEEE-Wiley, 2009. 